\title{
Inteligência e Desempenho Acadêmico: Revisão de Literatura
}

\author{
Felipe Valentini ${ }^{1}$ \\ Universidade Salgado de Oliveira - Universo, Niterói, Rio de Janeiro, Brasil \\ Jacob Arie Laros \\ Departamento de Psicologia Social e do Trabalho da Universidade de Brasília, \\ Brasilia, Distrito Federal, Brasil
}

\begin{abstract}
Resumo
A inteligência é um aspecto importante para a compreensão do desempenho acadêmico dos estudantes. Nesse contexto, buscou-se revisar a literatura recente sobre a relação entre a inteligência e o desempenho acadêmico, destacando as variáveis que podem influenciar essa relação. Os artigos revisados foram classificados em nove categorias segundo o conteúdo das variáveis que foram investigadas em relação à inteligência e ao desempenho acadêmico. Essas categorias referem-se às características de personalidade, sexo, cognição, etnia, aspectos positivos, disfuncionais, socioeconômicos, pessoais e aspectos da escola. Os artigos revisados indicam evidências de que as características de personalidade, como responsabilidade e autodisciplina, estão relacionadas, de maneira positiva, ao desempenho acadêmico. Outros aspectos como a criatividade, a motivação e a autopercepção de desempenho também podem explicar o desempenho. A revisão apresenta, ainda, as principais lacunas da literatura científica e as direções para as pesquisas futuras.
\end{abstract}

Palavras-chave: Inteligência, habilidades cognitivas, desempenho acadêmico.

\section{Intelligence and Academic Achievement: Literature Review}

\begin{abstract}
Intelligence is an important variable in the pursuit to comprehend academic achievement. The present study aimed to review the literature with regard to the relation between intelligence and academic achievement, highlighting variables that may influence this relation. The reviewed articles were classified into nine categories according to the main variables that have been investigated in relation to intelligence and academic achievement. These categories are related to personality traits, gender, cognition, ethnicity, positive and dysfunctional aspects, socioeconomic status, personal and school aspects. The reviewed articles indicate evidence that personality characteristics such as responsibility and self-discipline are positively associated with academic achievement. Other factors like creativity, motivation, self-perceived performance also seem to explain academic achievement. Furthermore, the main shortcomings of the scientific literature and directions for future research are presented.
\end{abstract}

Keywords: Intelligence, cognitive abilities, academic achievement.

Endereço para correspondência: Laboratório Meta, Sala A1-061/4, Universidade de Brasília, Campus Darcy Ribeiro, Brasília, DF, Brasil, 70910-900. E-mail: valentini.felipe@gmail.com e jalaros@gmail.com

Apoio e agradecimentos: O presente manuscrito foi desenvolvido com o apoio da Coordenação de Aperfeiçoamento de Pessoal de Nível Superior (CAPES). Os autores agradecem as importantes contribuições dos pesquisadores Dr. Ricardo Primi, Dra. Cláudia Cristina Fukuda, Dra. Juliana Barreiros Porto, Dra. Isolda Araújo Günther e Ms. Fabiana Damásio. 


\section{Inteligencia y Rendimiento Académico: Revisión de Literatura}

\section{Resumen}

La inteligencia es una variable importante para la comprensión del rendimiento de los estudiantes. El presente estudio tuvo como objetivo revisar la literatura reciente sobre la relación entre la inteligencia y el rendimiento académico, destacando las variables que pueden influir en esta relación. Los artículos revisados fueron clasificados en nueve categorías, de acuerdo a las variables que se han investigado en relación con la inteligencia y el rendimiento académico. Estas categorías se refieren a los rasgos de personalidad, sexo, cognición, etnia, aspectos positivos y disfuncionales, socioeconómicos, aspectos personales y de la escuela. Los artículos revisados indican una evidencia de que los rasgos de personalidad, como la responsabilidad y la autodisciplina, se asocian positivamente con el rendimiento académico. Otros factores como la creatividad, la motivación, el rendimiento autopercibida también pueden explicar el rendimiento académico. El estudio también presenta las principales deficiencias de la literatura científica y las direcciones para la investigación futura.

Palabras clave: Inteligencia, habilidades cognitivas, rendimiento académico.

As relações entre a inteligência e o desempenho acadêmico foram avaliadas por diversos estudos. Na maioria deles foi encontrada associação positiva com tamanho de efeito forte (Floyd, Evans, \& McGrew, 2003; Hattie, 2009; Primi, Ferrão, \& Almeida, 2010; Rohde \& Thompson, 2007). Embora essas relações estejam razoavelmente bem estabelecidas na literatura científica, ainda é necessário aprofundar o conhecimento especializado sobre quais as variáveis que influenciam a associação entre a inteligência e o desempenho de estudantes. Por exemplo, um professor, que leciona com alta proficiência de conteúdo, pode contribuir para o aumento no desempenho acadêmico de um estudante com habilidades cognitivas abaixo da média? Tais discussões poderiam ampliar a compreensão teórica sobre o fenômeno, bem como subsidiar políticas públicas eficientes.

A presente pesquisa insere-se nesse contexto e tem como objetivo revisar a literatura sobre as relações entre a inteligência e o desempenho acadêmico, principalmente nas disciplinas relacionadas à matemática e à linguagem. Buscou-se analisar os diversos aspectos apontados na literatura que podem influenciar, mediando ou moderando, as relações citadas. Primeiramente, serão apresentadas as principais definições e modelos teóricos de inteligência. Posteriormente, serão apresentados e discutidos os principais estudos empíricos e de meta-análise das relações entre a inteligência e o desempenho acadêmico.

No que se refere à definição de inteligência, ainda que não consensual, os principais conceitos englobam, de alguma forma, a capacidade para aprender relações, utilizando conhecimentos prévios ou apenas o raciocínio (Almeida, 1994). Essa compreensão é base para grande parte dos modelos fatoriais de inteligência, bem como para diversos instrumentos de avaliação psicológica. Para as baterias de inteligência Snijders Oomen (SON), por exemplo, o construto em questão é definido como a habilidade para aprender e a chance de sucesso na escola (Laros, Reis, \& Tellegen, 2010; Snijders, Tellegen, \& Laros, 1989). Ou seja, a própria definição da inteligência parece estar associada à capacidade de aprendizagem formal ou informal. Grande parte dos testes e estudos sobre a relação entre a inteligência e o desempenho acadêmico utilizou algum modelo fatorial de inteligência. Portanto, a presente revisão de literatura focará os modelos fatoriais.

Os modelos fatoriais buscam compreender apenas estrutura latente da inteligência. Em outras palavras, os teóricos dessa perspectiva estão mais interessados na taxionomia das dimensões da inteligência, normalmente avaliada por meio de estudos psicométricos, do que nos processos cognitivos subjacentes à dinâmica desse cons- 
truto. Nesse contexto, as proposições de Spearman e Thurstone são consideradas as primeiras teorias fatoriais. Ambos queriam responder à mesma pergunta: quantas e quais dimensões são necessárias para descrever, de forma eficiente e parcimoniosa, as habilidades cognitivas? Contudo, suas conclusões são bastante distintas (Hogan, 2006).

Spearman (1904) realizou estudos a partir de testes de funções sensoriais já existentes, buscando avaliar as correlações entre os testes. Por meio das análises dessas pesquisas, Spearman reuniu evidências de relações significativas entre todos os testes avaliados. Tais correlações, na visão do pesquisador, poderiam ser explicadas por uma dimensão latente subjacente aos testes específicos. Em outras palavras, Spearman concluiu que, embora os testes avaliassem habilidades cognitivas distintas, suas associações eram tão elevadas a ponto de sustentar a existência de uma 'habilidade geral', denominada fator $g$ (Brody, 2000; Hogan, 2006).

Thurstone (1938) discordava amplamente de Spearman. Segundo ele, uma dimensão não explicaria as especificidades das habilidades cognitivas. Thurstone desenvolveu um método de análise fatorial múltipla e, por meio desse método, concluiu que as correlações encontradas entre os testes não eram suficientemente altas para sustentar a existência de uma dimensão geral da inteligência (Brody, 2000; Davidson \& Downing, 2000; Thurstone, 1938).

As teorias de Spearman (1904) e Thurstone (1938) eram antagônicas e geraram inúmeras discussões sobre as dimensões teóricas da inteligência. Modelos posteriores ajudaram a conciliar as duas teorias. Um desses modelos foi teorizado por Cattell $(1943,1963)$, que organizou as habilidades cognitivas em duas dimensões gerais. A primeira delas, inteligência fluida (Gf), envolve as habilidades de raciocínio e a capacidade de solução de problemas novos. Nesse sentido, a inteligência fluida refere-se à capacidade de raciocinar indutiva e dedutivamente, formar e testar hipóteses para problemas novos e identificar relações e conceitos subjacentes às situações. A $G f$ é a dimensão que está mais associada ao fator $g$ de Spearman (Carroll, 2005; McGrew,
2005). A segunda dimensão, denominada inteligência cristalizada $(G c)$ está associada à aquisição e à solidificação dos conhecimentos formais e informais, aprendidos nas escolas ou por meio da transmissão cultural (Almeida, 1994; Cattell, 1943, 1963; Horn, 1994).

Modelos mais recentes incorporaram as dimensões $G f$ e $G c$ e ampliaram a compreensão da estrutura da inteligência. Um desses modelos foi desenvolvido por Carroll (1993, 2005). A partir de uma meta-análise de mais de 450 estudos, Carroll encontrou evidências que sustentavam uma organização hierárquica dos fatores da inteligência em três estratos. O estrato III é composto por uma grande habilidade geral. $\mathrm{O}$ estrato II agrega oito dimensões gerais: inteligência fluida, inteligência cristalizada, memória, percepção visual, percepção auditiva, habilidade de apreensão, velocidade cognitiva e velocidade de processamento. $\mathrm{O}$ estrato I é composto por habilidades mais específicas, tais como raciocínio quantitativo, compreensão de leitura, memória visual e tempo de reação. Ressalta-se que os três estratos organizam fatores de primeira, de segunda e de terceira ordem. Ou seja, todas as habilidades específicas do primeiro estrato estão relacionadas a alguma habilidade do segundo estrato. Além disso, as oito habilidades do estrato II podem ser explicadas, em grande parte, pelo fator geral do estrato III (Carroll, 1993, 2005).

Conforme indicado pelo próprio Carroll (2005), o modelo era provisório. Segundo o pesquisador, seria importante definir melhor as habilidades específicas do estrato I, bem como encontrar evidências da sua existência. Ademais, seria necessário aprofundar as discussões sobre as relações entre o estrato III e o fator $g$ de Spearman. Nesse contexto, McGrew e Flanagam (1998) propuseram uma reorganização e ampliação da teoria dos estratos por meio do modelo Cattell-Horn-Carroll (CHC). Na realidade, o modelo CHC, também organizado em uma estrutura de três níveis, é considerado uma integração da teoria dos três estratos e da teoria $G f-G c$. O nível mais amplo (fator de terceira ordem) é composto pela capacidade geral. No segundo nível, as habilidades são organizadas em 10 dimensões gerais: inteligência fluida, inteli- 
gência cristalizada, conhecimento quantitativo, leitura escrita, memória de curto prazo, processamento visual, processamento auditivo, capacidade da memória de longo prazo, velocidade do processamento e rapidez na decisão. Finalmente, o nível mais baixo é composto por aproximadamente 70 fatores bastante específicos, tais como raciocínio sequencial, raciocínio piagetiano, sensibilidade gramatical, proficiência em línguas estrangeiras e sensibilidade cinestésica. Os três níveis da estrutura do modelo $\mathrm{CHC}$ seguem uma ordem de especialização, variando do mais geral - nível III - até as dimensões específicas - nível I - (McGrew, 2005; Primi, 2003).

As dimensões estruturadas no modelo $\mathrm{CHC}$ já foram amplamente relacionadas, em pesquisas anteriores, ao desempenho acadêmico e à aprendizagem de estudantes. Rohde e Thompson (2007), por exemplo, investigaram o desempenho dos estudantes nas provas do sistema de avaliação educacional SAT (Scholastic Assessment Test) e do Teste de Desempenho Amplo (Wide Range Achievement Test [WRAT III]). O SAT é um exame educacional utilizado para ingresso nas universidades dos Estados Unidos (a proposta do SAT é semelhante ao ENEM [Exame Nacional do Ensino Médio], no Brasil). No estudo foram encontradas correlações moderadas e fortes $(0,30 \leq r \leq 0,71)$ entre os testes de desempenho e as avaliações das habilidades cognitivas realizadas por meio do teste Raven. Adicionalmente, quando controlados os efeitos da memória de trabalho, da velocidade de processamento e da habilidade espacial (avaliadas por uma bateria de testes e tarefas cognitivas), as habilidades cognitivas gerais continuaram acrescentando explicação para a variabilidade do desempenho acadêmico. Em outras palavras, ao remover os efeitos de outras variáveis cognitivas, os autores ainda encontraram relações significativas entre a inteligência geral e o desempenho acadêmico.

Um estudo de meta-análise foi conduzido por McGrew e Wendling (2010) sobre a relação entre o modelo Cattell-Horn-Carroll (CHC) de inteligência e o desempenho acadêmico. Embasados em 19 estudos anteriores, os pesquisadores concluíram que os fatores $g$ (geral) e $G f$ (inteligência fluida) relacionaram-se positivamente ao desempenho acadêmico em todos os estudos $(p<0,05)$.

Relações importantes entre a inteligência e o desempenho acadêmico também foram encontradas por Primi et al. (2010). Os autores utilizaram a Bateria de Provas de Raciocínio (BPR-5) para a avaliação da inteligência fluida e associaram-na a quatro medidas repetidas de desempenho em matemática, aplicadas entre os anos de 2005 e 2006. Em concordância com a maior parte da literatura científica, foram encontradas relações positivas entre o desempenho em matemática e as dimensões da inteligência geral, raciocínio abstrato, numérico, verbal e espacial ( $r \geq 0,27$, para a dimensão geral). Ademais, o maior crescimento (maior slope) na proficiência de matemática foi observado nos estudantes com os maiores escores de inteligência. Em outras palavras, além do desempenho mais elevado, os estudantes com altos escores de inteligência parecem adquirir mais conhecimento em matemática. Resultados semelhantes foram encontrados por Geary (2011). Esse autor conduziu um estudo longitudinal para observar o desempenho acadêmico de crianças do ensino fundamental. Para a avaliação da inteligência o autor utilizou a bateria WASI (Wechsler Abbreviated Scale of Intelligence). Os resultados indicaram que a inteligência e a velocidade de processamento previam a evolução da proficiência em matemática após cinco anos da realização da primeira avaliação. Ressalta-se ainda que a velocidade do processamento também é considerada uma dimensão específica da inteligência, segundo o modelo CHC.

As pesquisas, portanto, indicam relações moderadas e fortes entre a inteligência, o desempenho acadêmico e a aprendizagem. Contudo, ainda é necessário aprofundar a compreensão sobre quais variáveis do nível pessoal e do contextual poderiam influenciar a relação citada. Por exemplo, as características de personalidade podem aumentar ou diminuir a diferença do desempenho observada entre os estudantes com altos e baixos escores de inteligência? Quais outras variáveis são apontadas, em conjunto com a inteligência, como importantes para a compreensão do desempenho acadêmico? A presente pesquisa 
insere-se neste contexto e tem como objetivo revisar a literatura recente sobre a relação empírica entre a inteligência e o desempenho acadêmico, apontando as variáveis que podem moderar ou mediar essa relação.

\section{Método}

Para atingir o objetivo desta pesquisa, realizou-se, primeiramente, uma busca por artigos nas bases de dados do PsycInfo, Bireme, BVS-Psi, Pepsic e Scielo, publicados entre os anos de 2000 e 2012. Portanto, o período de aproximadamente 10 anos compreende apenas as publicações mais recentes sobre o tema. Justifica-se o recorte desse período em função da recenticidade das principais teorias da inteligência, principalmente do modelo CHC (que foi proposto no final da década de 1990). Para a recuperação dos artigos, foram utilizados os seguintes descritores: inteligência, desempenho acadêmico, matemática, linguagem, moderação e mediação.

As mais de 300 publicações recuperadas foram lidas pelos autores e por uma equipe de estudantes de psicologia. Contudo, uma parte significativa desses artigos não apresentava uma discussão condizente com os objetivos da presente revisão ou não tratavam de uma pesquisa empírica ou de meta-análise. Então, realizou-se uma seleção dos artigos recuperados, tendo como base os seguintes critérios de inclusão: (a) o artigo deveria apresentar dados de uma pesquisa empírica ou de meta-análise; (b) os testes de inteligência utilizados deveriam ser embasados em algum modelo fatorial da inteligência; (c) o desempenho acadêmico deveria ser mensurado por, ao menos, um teste de linguagem ou de matemática; (d) ao menos uma variável adicional deveria ter sido investigada, além da inteligência e do desempenho acadêmico. No que se refere à seleção dos artigos empíricos, não se adicionou nenhum critério de exclusão com base nas amostras de pesquisa. Portanto, foram selecionados todos os artigos empíricos, que avaliaram estudantes de todos os níveis educacionais, desde que tenha sido investigado o desempenho em provas de matemática ou linguagem. Com base nesses critérios, 50 artigos foram selecionados.
As 50 publicações selecionadas foram classificadas em categorias, segundo o conteúdo das variáveis que foram pesquisadas juntamente com a inteligência e o desempenho acadêmico. Em outras palavras, buscou-se classificar os tipos de variáveis que, em conjunto com a inteligência, podem explicar o desempenho acadêmico dos estudantes.

\section{Resultados}

Os artigos revisados foram classificados nas nove categorias apresentadas na Tabela 1. Cada categoria refere-se a um grupo de variáveis estudadas em relação à inteligência e ao desempenho acadêmico. Cabe destacar que os artigos poderiam avaliar mais de uma variável, de maneira concomitante. Caso as variáveis pesquisadas referiam-se a mais de uma categoria da Tabela 1, o artigo foi contabilizado em diferentes categorias. Por exemplo, a pesquisa de Steinmayr e Spinath (2008), investigou hipóteses complexas, que consideraram a relação entre a inteligência, o sexo e a personalidade na explicação do desempenho acadêmico. Portanto, tal pesquisa foi contabilizada tanto na categoria 'personalidade', quanto na categoria 'gênero/sexo'.

A seguir, as nove categorias da Tabela 1 serão discutidas. Para tanto, serão apresentados os resultados de artigos classificados em cada categoria. Essa apresentação não pretende ser exaustiva, mas apenas indicar os resultados mais relevantes em cada categoria.

Conforme a Tabela 1, uma das variáveis mais investigadas em relação à inteligência e ao desempenho acadêmico é a personalidade. Fabio e Busoni (2007), por exemplo, desenvolveram um modelo, a partir dos dados coletados com o teste Raven e o Questionário Big Five (BFQ). Além da inteligência, os resultados indicaram que o fator de personalidade Realização (ou Conscienciosidade) é um preditor dos exames finais de estudantes do ensino secundário italiano. No modelo final, a inteligência explicou $17 \%$ da variância do desempenho acadêmico, e os traços de personalidade acrescentaram mais $11 \%$ de explicação. Ou seja, características como perseverança, senso de dever e autodisciplina (asso- 


\section{Tabela 1}

Categorias das Variáveis Associadas à Inteligência e ao Desempenho Acadêmico

\begin{tabular}{lcc}
\hline Categorias & Descrição & Número \\
& de artigos \\
\hline
\end{tabular}

1. Personalidade Estudos que consideraram as características de personalidade, em grande parte associadas ao modelo dos Cinco Grandes Fatores de Personalidade.

2. Gênero/Sexo Estudos que consideraram as características relacionadas à identidade de gênero ou ao sexo do participante.

3. Outros Aspectos Estudos que avaliaram aspectos cognitivos, além da inteligência, tais como Cognitivos atenção, criatividade, velocidade de processamento, memória e funções executivas.

4. Etnia/Cor de pele Estudos que avaliaram a influência da cor da pele do participante ou do grupo étnico sobre o desempenho acadêmico.

5. Aspectos

Positivos

Aspectos normalmente associados à psicologia positiva tais como motivação, senso de competência e pensamentos positivos sobre a capacidade de alcançar os objetivos planejados.

6. Aspectos

Disfuncionais

Aspectos negativos, normalmente associados a disfuncionalidades ou à psicopatologia, tais como estresse, baixa autoestima, faltas escolares e expulsão da escola.

7. Aspectos

Aspectos contextuais relacionados ao acesso a recursos econômicos, renda,

Socioeconômicos escolaridade dos pais e ocupação dos pais.

8. Outros

Aspectos Pessoais

Diversos aspectos do nível pessoal que não foram abarcados pelas categorias anteriores, tais como qualidade das interações sociais, qualidade do sono, diferenças entre estudantes que produzem mais no período da manhã ou à noite, experiência de trabalho e desempenho de crianças adotadas.

9. Aspectos

Variáveis do nível de análise da escola, tais como dever de casa, efeito de programas pré-escolares e anos de permanência nas escolas.

ciadas ao fator Realização) foram relacionadas positivamente ao desempenho acadêmico.

Estudo semelhante ao de Fabio e Busoni (2007) foi conduzido por Rosander, Bäcktröm e Stenberg (2011). Esses últimos autores utilizaram o teste WPT (Wonderlic Personnel Test) para avaliar o fator geral de inteligência de crianças suecas e, para a análise de dados, a modelagem por equações estruturais. As conclusões indicaram que, além da inteligência e do fator de personalidade Realização, os fatores Extroversão e Neuroticismo associaram-se significativamente às provas de linguagem, ciências sociais, matemática, artes e esportes. O fator Neuroticismo teve um impacto positivo (coeficiente padronizado $=0,14$ ), e a Extroversão, negativo (coeficiente padronizado $=-0,14$ ).
Portanto, as características de assertividade, festividade e gregarismo (fator Extroversão), bem como a instabilidade emocional (fator Neuroticismo) também estão associadas ao desempenho acadêmico. Os autores argumentaram que, provavelmente, ambos os fatores estejam influenciando a motivação para o estudo e, indiretamente, o desempenho acadêmico.

As direções das relações encontradas por Rosander et al. (2011) foram semelhantes às apresentadas por Furnham e Monsen (2009), que investigaram a relação entre a inteligência, a personalidade e as notas de inglês (linguagem), literatura, matemática, ciências e outras disciplinas eletivas de adolescentes britânicos. Para a avaliação do fator geral de inteligência, os autores utilizaram o teste WPT (Wonderlic 
Personnel Test). Além da relação positiva entre a inteligência e o desempenho acadêmico (Beta =0,34), Furnham e Monsen também encontraram associação negativa entre o fator de personalidade Extroversão e a nota geral das provas escolares $($ Beta $=-0,18)$. Todavia, quando considerado apenas o desempenho nas disciplinas eletivas (Francês, Geografia e Alemão), a personalidade Extrovertida não apresentou relação estatisticamente significativa com o desempenho acadêmico.

A segunda categoria apresentada na Tabela 1 diz respeito aos estudos que avaliaram a variável sexo ou a variável gênero, além da inteligência, relacionada ao desempenho acadêmico. Ainda que a literatura científica apresente um número considerável de pesquisas empíricas, os resultados apontam para diferentes conclusões. Kuhn e Holling (2009) avaliaram o desempenho de aproximadamente 1.100 estudantes alemães. Os autores utilizaram o teste BIS (Berlin Structure of Intelligence) para a avaliação da inteligência. $O$ teste BIS agrega subtestes de raciocínio e subtestes de velocidade de processamento. Os autores concluíram que as meninas obtiveram desempenho maior nas provas de linguagem (tamanho de efeito $d$ de Cohen $=0,24$ ), e os meninos, nas provas de ciências $(d=0,13)$. Especificamente para as meninas, o desempenho em linguagem continua alto, após controlar o efeito da inteligência. Kaufman, Kaufman, Liu e Johnson (2009) também encontraram um efeito do gênero sobre o desempenho de adultos americanos. Neste estudo, os escores das inteligências fluida e cristalizada foram mensurados por meio do teste KBIT. As mulheres obtiveram um desempenho maior nas provas de escrita, e os homens, nas provas de matemática. Ademais, não foram encontradas diferenças estatisticamente significativas entre homens e mulheres no que se refere à inteligência fluida e à cristalizada.

Ainda no que se refere ao gênero e ao sexo, a literatura é um pouco controversa. Por um lado, no estudo de Furnham e Mosen (2009), as meninas obtiveram um desempenho superior nas provas de história e tecnologia, e os meninos, nas provas de literatura, matemática e ciências. Ademais, a combinação entre a inteligência, a personalidade e o sexo explicou mais de $25 \%$ da variância do desempenho nas diferentes provas. Os resultados do estudo de Steinmayr e Spinath (2008) também indicaram a associação entre a inteligência, o sexo e o desempenho acadêmico. Neste estudo, os autores utilizaram o teste IST (Intelligence Structure Test) para a avaliação da inteligência. Adicionalmente, os autores apresentaram a característica de personalidade agradabilidade como mediadora da relação entre o sexo e o desempenho. Por outro lado, os resultados do estudo de Leclerc, Larivée, Archambault e Janosz (2010) indicaram que o sexo não estava relacionado ao desempenho acadêmico, tampouco influenciava a relação entre a inteligência e as crenças de autocompetência. Estes últimos autores utilizam o teste de Matrizes Progressivas de Raven.

Outro conjunto de variáveis estudadas em relação ao desempenho diz respeito aos aspectos cognitivos (além da inteligência), descritos na terceira categoria da Tabela 1. Nesse aspecto, a criatividade parece exercer um papel importante na explicação do desempenho acadêmico. A criatividade pode ser definida como a fluidez e a flexibilidade na produção de ideias originais. Rindermann e Neubauer (2004) testaram um modelo teórico, por meio de equações estruturais, cuja variável dependente era o desempenho acadêmico em linguagem, matemática, física e ciências humanas. A despeito do grande efeito da inteligência, avaliada por meio do teste Raven, (coeficiente padronizado $=0,53$ ), a criatividade também influenciou o desempenho acadêmico dos estudantes (coeficiente padronizado $=0,19$ ). Resultados semelhantes ao estudo de Rindermann e Neubauer foram encontrados por Freund, Holling e Preckel (2007). Esses autores investigaram 1.500 estudantes alemães no que se refere às habilidades cognitivas (avaliadas por meio do teste BIS [Berlin Structure of Intelligence]), ao desempenho acadêmico e à criatividade, entre outros aspectos. Por meio de análises de regressão multinível, os autores encontraram um impacto significativo da inteligência sobre o desempenho acadêmico (Beta $=$ 0,47 , para ciências naturais; e Beta $=0,16$, para as ciências sociais). Além disso, a criatividade 
também se associou ao desempenho, principalmente na área de ciências sociais $($ Beta $=0,23)$. Entretanto, o impacto da criatividade sobre o desempenho em matemática e ciências naturais foi menor $($ Betas $=0,10)$.

Ainda no que se refere aos aspectos cognitivos, Swanson, Jerman e Zheng (2008) investigaram a capacidade de crianças na solução de problemas matemáticos. Os resultados indicaram que a inteligência, avaliada por meio do teste Matrizes Coloridas de Raven, e a memória de trabalho foram variáveis preditoras da solução de problemas matemáticos. Outra variável cognitiva importante diz respeito à atenção. $\mathrm{O}$ estudo de Steinmayr, Ziegler e Träuble (2010) apontou para a qualidade da atenção sustentada na predição do desempenho escolar, além da inteligência (mensurada pelo teste Intelligence Structure Test [IST]). Cabe destacar ainda o papel das funções executivas, que estão associadas aos processos cognitivos responsáveis pelo planejamento e execução de atividades. Nesse contexto, a pesquisa de Clark, Pritchard e Woodward (2010) encontrou evidências da explicação do desempenho acadêmico de crianças por meio das funções executivas de flexibilidade e de controle inibitório, além da inteligência avaliada por meio do teste WPPSI-R (Wechsler Preschool and Primary Scale of Intelligence).

A quarta categoria de variáveis estudadas em relação ao desempenho acadêmico diz respeito à etnia e à cor de pele (Tabela 1). Frederickson e Petrides (2008), por exemplo, investigaram uma amostra de aproximadamente 500 estudantes britânicos. O desempenho dos adolescentes britânicos brancos foi superior se comparado ao dos estudantes britânicos negros, bem como superior aos estudantes descendentes de paquistaneses (tamanhos de efeito $d$ de Cohen entre 0,08 e 0,13$)$. No entanto, quando controlado o efeito da variável inteligência, as diferenças entre os grupos foram minimizadas ou não apresentaram significância estatística. Nesta pesquisa, os autores avaliaram a inteligência cristalizada por meio do teste VRT (Verbal Reasoning Test). Conclusões semelhantes foram encontradas por Swartwout, Garnaat, Myszka, Fletcher e Dennis (2010). Para avaliação da inteligência os autores utilizaram os testes Stanford Binet IV e Woodcock-Johnson. Os resultados desse estudo indicaram que as crianças com o nível socioeconômico baixo e descendentes de hispânicos obtiveram desempenho menor em provas verbais (em inglês), se comparadas às crianças brancas não hispânicas. Todavia, para as provas não verbais, o desempenho das crianças hispânicas praticamente se igualou ao das crianças brancas não hispânicas. Em outras palavras, as diferenças do desempenho dos estudantes se devem, provavelmente, às questões associadas à linguagem e não à etnia ou à cor de pele.

A quinta categoria destacada na Tabela 1 refere-se aos aspectos psicológicos positivos associados ao desempenho acadêmico. Tais aspetos dizem respeito ao papel das variáveis que contribuem para uma vida saudável. Day, Hanson, Maltiby, Proctor e Wood (2010), por exemplo, estudaram a influência da variável esperança na relação entre a inteligência e o desempenho acadêmico. A esperança, nesse contexto, foi definida como uma característica da personalidade associada aos pensamentos positivos sobre a capacidade de alcançar os objetivos planejados. Os autores mensuraram o fator geral de inteligência por meio do teste Raven. Por meio de uma regressão hierárquica, os autores evidenciaram que o fator esperança, além da personalidade e da inteligência, associou-se ao desempenho acadêmico. Para a inteligência e para a personalidade, os valores de Beta (da análise de regressão linear) variaram de 0,03 a 0,34 ; e, para a esperança, os Betas variaram de 0,16 e 0,30 . Ou seja, a crença de que é possível criar planos de sucesso para alcançar um objetivo específico ou resolver um problema mostrou-se associada ao desempenho acadêmico superior.

Dentro do contexto da psicologia positiva, Leclerc et al. (2010) estudaram a relação entre a inteligência (mensurada pelo Raven), as crenças de autocompetência e o desempenho acadêmico. Segundo os autores, a autocompetência se refere à autopercepção das próprias habilidades e das capacidades escolares. Os autores avaliaram aproximadamente 900 estudantes universitários canadenses francófonos. Por meio de correlações e de modelos de regressão, Leclerc et 
al. (2010) concluíram que, além da inteligência $($ Beta $=0,07)$, a autocompetência relacionou-se positivamente e de maneira forte com o desempenho acadêmico $($ Beta $=0,79)$. Ademais, o fator de interação entre as variáveis inteligência e autocompentência apresentou predição positiva sobre o desempenho nas escolas $($ Beta $=0,08)$. Em outras palavras, a relação entre a inteligência e o desempenho acadêmico é mais forte para os estudantes com altos escores de autocompetência.

Ainda no contexto da psicologia positiva, Leeson, Ciarrochi e Heaven (2008) estudaram, entre outros aspectos, a relação entre o pensamento positivo, a inteligência e o desempenho acadêmico. Os pensamentos positivos dizem respeito às características pessoais de autoestima, estilos atribucionais e esperança. Durante o estudo, os autores investigaram 784 estudantes universitários australianos, que responderam, entre outros instrumentos, a uma bateria de subtestes para avaliar a inteligência verbal e numérica. O modelo testado indicou que a inteligência e o pensamento positivo associaram-se ao desempenho acadêmico (coeficientes padronizados de 0,50 e 0,13, respectivamente). Especificamente, a esperança e os estilos atribucionais são preditores mais fortes do desempenho acadêmico se comparados à autoestima.

Ainda no que se refere categoria de variáveis positivas, a motivação foi objeto de estudo de Steinmayr e Spinath (2009). Os autores avaliaram a inteligência (mensurada pelo teste IST [Intelligence Structure Test]) e a proficiência em matemática e linguagem de estudantes alemães. No modelo de regressão do desempenho acadêmico foram apresentados os valores de Beta para os preditores inteligência $(0,08)$, esperança de sucesso $(0,04)$, medo de falhar $(-0,05)$ e competitividade $(0,07)$. Portanto, uma parte do desempenho acadêmico pode ser explicada pelos aspectos relacionados à motivação acadêmica do estudante.

A autopercepção do desempenho também é umaspectopositivo(daquinta categoria da Tabela 1) que merece destaque. Chamorro-Premuzic, Harlaar, Greven e Plomin (2010) conduziram um estudo longitudinal com gêmeos britânicos para investigar a autopercepção das habilidades cognitivas. Além da inteligência (avaliada por meio do WISC-III), a autopercepção também apresentou relação positiva com o desempenho acadêmico (coeficientes padronizados entre 0,11 e 0,41). Além disso, o impacto do desempenho acadêmico na primeira avaliação $\left(\mathrm{DA}_{1}\right)$ sobre a autopercepção de desempenho na segunda avaliação $\left(\mathrm{APD}_{2}\right)$ foi semelhante ao impacto do efeito da autopercepção na primeira avaliação $\left(\mathrm{APD}_{1}\right)$ sobre o desempenho na segunda $\left(\mathrm{DA}_{2}\right)$ $\left[\mathrm{DA}_{1} \rightarrow \mathrm{APD}_{2} \approx \mathrm{APD}_{1} \rightarrow \mathrm{DA}_{2}\right.$, coeficientes padronizados de 0,13 e 0,11 , respectivamente]. Ou seja, o desempenho e a autopercepção se influenciaram mutuamente dentro de um sistema de retroalimentação. Diante disso, os autores afirmam que tais relações podem ser explicadas pela autoeficácia e pelo insight: o alto desempenho poderia aumentar a autopercepção do desempenho (insight), que, por sua vez, poderia retroalimentar o sistema, aumentado o desempenho.

Por outro lado, a sexta categoria de variáveis (Tabela 1) diz respeito às características pessoais negativas, normalmente relacionadas às psicopatologias. Nesse contexto, Freudenthaler, Spinath e Neubauer (2008) pesquisaram a ansiedade escolar e a autoestima numa amostra de 1.353 estudantes austríacos. Os autores mensuraram a inteligência numérica, verbal e visoespacial por meio do teste ISA (Intelligenz Struktur Analyse). Os resultados indicaram que a ansiedade escolar (que está relacionada à preocupação com o desempenho na escola) associou-se ao desempenho negativo dos meninos (Beta de regressão $=-0,11$ ). Ademais a autoestima associou-se positivamente ao desempenho dos meninos e das meninas (respectivos Betas, 0,13 e 0,23).

Ainda no que se refere às características negativas, Petrides, Frederickson e Furnham (2004) associaram o desempenho acadêmico à inteligência emocional e ao comportamento escolar desviante. $\mathrm{O}$ modelo explicativo criado pelos autores indicou que o baixo desempenho escolar pode ser explicado por meio da suscetibilidade às pressões acadêmicas apresentadas por pessoas com baixos escores de inteligência emocional. 
Outro aspecto importante a ser considerado na relação entre a inteligência e o desempenho acadêmico diz respeito ao nível socioeconômico (sexta categoria da Tabela 1). O nível socioeconômico (NSE) foi estudado por Colom e Flores-Mendonza (2007) numa amostra de 640 estudantes de diferentes escolas brasileiras. Nessa pesquisa, a inteligência foi mensurada por meio dos testes Raven e WISC-III, e o NSE foi estudado por meio do salário familiar e nível educacional dos pais. Os resultados indicaram que o NSE não foi capaz de predizer as diferenças no desempenho acadêmico $($ Beta $=0,04)$. Ademais, a inteligência se mostrou como o único preditor das notas escolares (Beta $=0,37)$. Conclusões semelhantes foram encontradas por Frederickson e Petrides (2008) numa pesquisa com aproximadamente 500 adolescentes britânicos. Os resultados apontaram para uma diminuição do efeito do NSE sobre o desempenho acadêmico após o controle da variável inteligência (avaliada pelo teste VRT [Verbal Reasoning Test]).

Entretanto, é necessário ressaltar que alguns estudos, que não consideraram a variável inteligência, apresentaram relações significativas entre o NSE e o desempenho acadêmico. Ao considerar o nível de análise da escola, Laros, Marciano e Andrade (2010) evidenciaram que a inserção do NSE (agregado para a escola) explicou $77 \%$ da variância do desempenho acadêmico (do nível da escola). Em outro estudo, os mesmos autores apresentaram um modelo capaz de explicar aproximadamente $70 \%$ da variância do desempenho em língua portuguesa, por meio das variáveis NSE agregado para a escola, escolaridade da mãe e etnia do aluno (Laros, Marciano, \& Andrade, 2012).

Ressalta-se, ainda, a relação entre o NSE e a inteligência evidenciada pelas pesquisas que não consideraram o desempenho acadêmico. Laros, Tellegen, Jesus e Karino (no prelo), por exemplo, encontram correlações moderadas e fortes entre os testes de inteligência SON-R e WPPSI-III e o NSE ( $r$ entre 0,40 e 0,67$)$. As correlações mais altas referiam-se aos testes verbais de inteligência. Nesse sentido, os aspectos associados ao desenvolvimento do vocabulário e de conhecimentos gerais podem depender mais do acesso à cultura e à educação do que os aspectos relacionados ao raciocínio abstrato e à solução de problemas para os quais não é necessário o conhecimento prévio. Em outras palavras, o desempenho em tarefas de inteligência fluida depende menos do NSE, se comparado às tarefas que exigem conhecimento prévio.

A oitava categoria descrita na Tabela 1 agrega uma miscelânea de variáveis do nível de análise do indivíduo que não corresponde a nenhuma das sete categorias anteriores. Por exemplo, a meta-análise de Preckel, Lipnevich, Schneider e Roberts (2011) indicou que o cronótipo matutino (pessoas que produzem mais durante o período da manhã) esteve positivamente relacionado ao desempenho acadêmico $(r=0,16)$ e negativamente à inteligência $(r=-0,04)$. Destaca-se ainda, dentro da oitava categoria, a qualidade do sono na predição do desempenho acadêmico. Johnston, Gradisar, Dohnt, Billows e Mccappin (2010) pesquisaram uma amostra de aproximadamente 200 adolescentes e concluíram que a dificuldade do sono explicou 2,9 \% da variância do desempenho acadêmico. Contudo essa relação diminuiu após o controle da variável inteligência (mensurada por meio de uma série de testes construídos para avaliar a inteligência fluida).

Finalmente, a nona categoria da Tabela 1 refere-se às variáveis relacionadas à escola. A pequisa de Kaufman et al. (2009) evidenciou o papel da escola no desempenho acadêmico. Os resultados do estudo indicaram que a inteligência fluida e a inteligência cristalizada ( $G f$ e $G c$ ), mensuradas pelo KBIT, estão relacionadas ao número de anos que o aluno permanece nas escolas formais. Além disso, o número de anos na escola associou-se ao desempenho nas disciplinas de matemática, leitura e escrita. Para a disciplina de matemática, essa relação foi mais forte $(r=0,63)$ do que para as demais disciplinas $(r=$ $0,48$ e 0,49$)$. Em outras palavras, o desempenho em matemática parece depender mais da quantidade de anos nos quais a criança permanece na escola do que o desempenho em leitura e escrita.

Ainda no contexto da escola, Gorey (2001) conduziu um estudo meta-analítico para avaliar os efeitos dos programas pré-escolares ao longo da vida dos estudantes. Os autores utilizaram 
os dados de mais de 30 artigos e compararam as crianças que sofreram a intervenção com as crianças do grupo controle. Os programas pré-escolares apresentaram, na grande maioria dos estudos, um efeito positivo e forte sobre a inteligência e o desempenho acadêmico (médias dos tamanhos do efeito $U=76,5$ e 78,2 , respectivamente). Depois de cinco anos da intervenção, aproximadamente $70 \%$ das crianças que participaram dos programas ainda obtiveram desempenho melhor do que o grupo controle.

\section{Discussão e Direções para Pesquisas Futuras}

De maneira geral, as pesquisas, com objetivos e contextos diversos, apontam para a inteligência como uma importante variável para a compreensão do desempenho acadêmico. Nesse sentido, a variável inteligência parece explicar uma parte importante do desempenho em linguagem e matemática, mesmo quando removidos os efeitos de outras variáveis cognitivas, tais como velocidade do processamento e memória de trabalho (Rohde \& Thompson, 2007). Ademais, a inteligência parece influenciar também o aumento da proficiência acadêmica no decorrer dos anos (Primi et al., 2010). Assim, as crianças e os adolescentes com escores elevados na inteligência fluida tendem a apresentar aumento mais acentuado no desempenho. Em outras palavras, a aprendizagem também parece ser influenciada pelas habilidades cognitivas.

No entanto, cabe destacar que as pesquisas revisadas utilizaram diferentes instrumentos para a avaliação da inteligência, cujos modelos teóricos podem apresentar algumas diferenças. A ausência de controle refinado dessas diferenças constitui uma limitação desta revisão teórica, visto que os tamanhos dos efeitos indicados podem estar associados ao conceito psicológico utilizado pelo instrumento. Portanto, sugerimos que o leitor tenha cautela ao comparar os tamanhos de efeito apresentados. Contudo, é possível sustentar, ao menos teoricamente, que todos os estudos revisados avaliaram a inteligência. Ressaltamos que a organização hierárquica do modelo CHC indica que as correlações entre as dimensões específicas podem ser explicadas por uma dimensão geral. Além disso, as variâncias residuais dos fatores de segunda ordem não são correlacionadas entre si. Portanto, ainda que os instrumentos possam avaliar diferentes aspectos da inteligência, eles mantêm uma dimensão comum capaz de explicar uma parte significativa das variâncias das dimensões específicas.

No que se refere às variáveis investigadas em relação à inteligência e ao desempenho acadêmico, as características de personalidade foram as que apresentaram o maior número de artigos publicados, conforme a Tabela 1. Por um lado, características de autodisciplina, de senso de dever e de perseverança (fator Realização) associam-se positivamente ao desempenho. Por outro lado, características de festividade, de gregarismo e de assertividade (fator Extroversão) associam-se negativamente ao desempenho. Todavia, as pesquisas utilizam, normalmente, como métodos de análise, correlação e regressão linear. Consequentemente, a personalidade é estudada simplesmente como uma variável independente que, ao lado da inteligência, explica o desempenho acadêmico.

Ainda que a relação entre a personalidade e o desempenho acadêmico tenha sido amplamente investigada por estudos anteriores, é necessário aumentar as evidências da moderação e/ ou mediação que a personalidade exerce sobre a relação entre a inteligência, o desempenho e a aprendizagem. Nesse sentido, seria importante avaliar se as características associadas principalmente aos fatores Realização e Extroversão associam-se à relação entre a inteligência e o aumento da proficiência acadêmica ao longo dos anos. Em outras palavras, as características de personalidade podem aumentar ou diminuir a diferença da aprendizagem entre os estudantes com maiores e menores escores de inteligência? Para responder a esta pergunta, são necessários novos estudos com delineamentos longitudinais.

No que diz respeito às variáveis sexo e gênero, a literatura não apresenta evidências suficientes para uma conclusão robusta. Diversos estudos foram apresentados e apontam para diferenças no desempenho entre meninos e meninas. Contudo, quando controlado o efeito da variável 
inteligência a relação entre o sexo e o desempenho não é tão clara, podendo apresentar efeito significativo (Kaufman et al, 2009) ou não significativo (Leclerc et al., 2010).

No que se refere às variáveis cognitivas, a criatividade, a memória de trabalho, a atenção sustentada e as funções executivas associaram-se de maneira positiva ao desempenho acadêmico, mesmo após o controle da variável inteligência. Especificamente no que se refere à criatividade, a influência dessa variável parece mais relevante para as disciplinas associadas às ciências humanas, em detrimento às ciências naturais (Freund et al., 2007). Segundo os autores, é possível que as respostas criativas dos estudantes sejam mais valorizadas pelas áreas menos técnicas, cuja estrutura do conhecimento seja mais aberta e flexível. Essa hipótese explicaria a diferença do impacto da criatividade sobre o desempenho acadêmico. Em resumo, os estudos apontam para a importância de considerar os diversos aspectos cognitivos, além da inteligência, na relação com o desempenho acadêmico. Entretanto, ainda é necessário aprofundar o conhecimento sobre como esses processos cognitivos interagem entre si e com a inteligência, e como essa interação pode contribuir para a compreensão do desempenho acadêmico de uma maneira mais complexa. Sugere-se, portanto, a condução de pesquisas multivariadas que testem, por meio da modelagem por equações estruturais, a interação entre os processos cognitivos de atenção, memória, criatividade, funções executivas e inteligência, e a sua capacidade de explicação do desempenho acadêmico.

As variáveis etnia, cor de pele e nível socioeconômico (NSE), descritas nas quarta e sétima categoria da Tabela 1, parecem apresentar uma relação complexa com o desempenho acadêmico. As relações são fortes ao considerar apenas as variáveis NSE e inteligência (Laros et al., no prelo). Relações fortes também foram encontradas pelas pesquisas que consideraram apenas as variáveis NSE e desempenho acadêmico, principalmente para o nível de análise da escola (Laros, Marciano, et al., 2010, 2012). Contudo, ao controlar o efeito da inteligência, o impacto do NSE sobre o desempenho diminui considera- velmente ou torna-se não significativo (Colom \& Flores-Mendoza, 2007). Para a variável cor de pele, as conclusões são semelhantes: quando controlado o efeito da variável inteligência, as diferenças de desempenho entre pessoas de cor de pele ou etnias distintas também são bastante atenuadas. Esses resultados parecem indicar que se o NSE e a cor de pele forem, de fato, relevantes para a compreensão do desempenho acadêmico, o impacto deles é complexo, estrutural e podem influenciar, inclusive, o desenvolvimento das capacidades cognitivas de raciocínio e de resolução de problemas. Nesse sentido, ressalta-se ainda mais a importância de investigar esses aspectos por meio de pesquisas com delineamentos experimentais e longitudinais, bem como por meio de equações estruturais multinível.

No que se refere às características psicológicas positivas e negativas, descritas nas categorias cinco e seis, a autopercepção de desempenho, a motivação, os pensamentos positivos parecem contribuir positivamente para o desempenho acadêmico; entretanto a ansiedade, a baixa autoestima e a suscetibilidade às pressões acadêmicas associaram-se de maneira negativa ao desempenho. Considerando os tamanhos de efeito apesentados nos artigos revisados, os aspectos positivos parecem contribuir mais para a explicação do desempenho acadêmico do que os aspectos negativos. Especificamente sobre a autopercepção de desempenho, Chamorro-Premuzic et al. (2010) indicam evidências de um sistema de retroalimentação: a autopercepção elevada de desempenho tende a aumentar o real desempenho, que, por sua vez, reforça e aumenta a autopercepção. Ressalta-se, no entanto, que o estudo utilizou a inteligência apenas como uma variável de controle. Pesquisas que avaliem o efeito de moderação e/ou mediação da inteligência poderiam ampliar a compreensão desse sistema.

Alguns estudos revisados apresentaram resultados de relações entre o desempenho acadêmico, a inteligência e diversas variáveis isoladas que não puderam ser agrupadas em nenhuma das categorias anteriores. Ainda que tais estudos tenham apresentado relações estatisticamente significativas com o desempenho acadêmico, prin- 
cipalmente no que se refere à qualidade do sono e ao cronótipo matutino, a pequena quantidade de pesquisas não permite conclusões robustas.

Além das características específicas do contexto estudante (discutidas nas categorias de 1 a 8), a escola parece desempenhar papel importante no desempenho dos estudantes, conforme categoria nove da Tabela 1. Crianças com maiores escores de inteligência permanecem mais tempo na escola e apresentam melhor desempenho, principalmente em matemática (Gorey, 2001; Kaufman et al., 2009). Entretanto, é necessário aprofundar os estudos sobre quais aspectos específicos da instituição influenciam a relação entre a inteligência e o desempenho. Nesse contexto, é relevante investigar, por exemplo, se os professores mais bem preparados teórica e didaticamente influenciam fortemente o desempenho dos estudantes (principalmente daqueles com baixos escores de inteligência). Cabe destacar ainda que os artigos revisados, normalmente, apresentaram os resultados em apenas um nível de análise. Contudo, as características da escola, a inteligência e o desempenho dos estudantes são características de níveis de análise distintos. Portanto, sugere-se que novas pesquisas investiguem a relação entre a inteligência, as variáveis da escola (tais como, infraestrutura, qualidade dos professores e projetos pedagógicos, entre outras) e o desempenho acadêmico por meio de análises multinível, separando as variâncias por nível de análise.

Como principal limitação desta revisão teórica, cita-se a recuperação dos artigos em uma limitada quantidade de bases de dados. Essa limitação refere-se, principalmente, à dificuldade para recuperar as publicações reunidas nas bases de dados pagas e não disponibilizadas pela $\mathrm{Ca}$ pes. Consequentemente, a presente revisão pode sub ou superestimar o número de estudos e a importância das variáveis estudadas em relação ao desempenho acadêmico e à inteligência.

Em resumo, a presente revisão de literatura apresentou diversas variáveis que foram estudadas em relação à inteligência e ao desempenho acadêmico. Na maior parte das pesquisas, tais variáveis foram investigadas apenas como explicativas do desempenho. Contudo, é possível que esses fenômenos sejam mais complexos e precisem de modelos explicativos mais complexos. Portanto, a partir desta revisão, propõe-se uma agenda de pesquisa na qual as variáveis dos níveis do estudante sejam investigadas como mediadoras e/ou moderadoras do efeito da inteligência sobre o desempenho acadêmico. Ademais, é importante avaliar se tais variáveis influenciam o aumento do desempenho acadêmico no decorrer dos anos (ou seja, a aprendizagem). Espera-se, também, que os aspectos da escola, como a infraestrutura e o desempenho dos professores (relacionado ao conteúdo e à didática) também possam moderar a relação entre inteligência e o desempenho dos estudantes.

\section{Referências $^{2}$}

Almeida, L. S. (1994). Inteligência: Definição e medida. Aveiro, Portugal: CIDInE.

Brody, N. (2000). History of theories and measurements of intelligence. In R. J. Sternberg (Ed.), Handbook of intelligence (pp. 16-33). New York, NY: Cambridge University Press.

Carroll, J. B. (1993). Human cognitive abilities: A survey of factor-analytic studies. Cambridge, UK: Cambridge University Press.

Carroll, J. B. (2005). The three-stratum theory of cognitive abilities. In D. P. Flanagan \& P. L. Harrison (Eds.), Contemporary intellectual assessment ( $2^{\text {nd }}$ ed., pp. 69-76). New York, NY: The Guilford Press.

Cattell, R. B. (1943). The measurement of adult intelligence. Psychological Bulletin, 40, 153-193.

Cattell, R. B. (1963). Theory of fluid and crystallized intelligence: A critical experiment. Journal of Educational Psychology, 54, 1-22.

Chamorro-Premuzic, T., Harlaar, N., Greven, C. U., \& Plomin, R. (2010). More than just IQ: A longitudinal examitaion of self-perceived abilities as predictors of academic performance in a large sample of UK twins. Intelligence, 38, 385-392. *

Clark, C. A. C., Pritchard, V. E., \& Woodward, L. J. (2010). Preschool executive functioning abilities predict early mathematics achievement. Develo-

As publicações assinaladas com asterisco (*) fazem parte do grupo de artigos revisados. 
pmental Psychology, 5, 1176-1191. *

Colom, R., \& Flores-Mendoza, C. E. (2007). Intelligence predicts scholastic achievement irrespective of SES factors: Evidence from Brazil. Intelligence, 35, 243-251. *

Davidson, J. E., \& Downing, C. L. (2000). Contemporary models of intelligence. In R. J. Sternberg (Ed.), Handbook of intelligence (pp. 34-52). New York, NY: Cambridge University Press.

Day, L., Hanson, K., Maltby, J., Proctor, C., \& Wood, A. (2010). Hope uniquely predicts objective academic achievement above intelligence, personality, and previous academic achievement. Journal of Research in Personality, 44, 550-553. *

Fabio, A. D., \& Busoni, L. (2007). Fluid intelligence, personality traits and scholastic success: Empirical evidence in a sample of Italian high school students. Personality and Individual Differences, 43, 2095-2104. *

Floyd, R. G., Evans, J. J., \& McGrew, K. S. (2003). Relations between measures of Cattell-Horn-Carroll (CHC) cognitive abilities and mathematics across the school-age years. Psychology in the Schools, 40, 155-171.

Freudenthaler, H., Spinath, B., \& Neubauer, A. C. (2008). Predicting school achievement in boys and girls. European Journal of Personality, 22, 231-245. *

Frederickson, N., \& Petrides, K. V. (2008). Ethnic, gender, and socio-economic group differences in academic performance and secondary school selection: A longitudinal analysis. Learning and Individual Differences, 18, 144-151. *

Freund, P. A., Holling, H., \& Preckel, F. (2007). A multivariate, multilevel analysis of the relationship between cognitive abilities and scholastic achievement. Journal of Individual Differences, 28, 188-197. *

Furnham, A., \& Monsen, J. (2009). Personality traits and intelligence predict academic school grades. Learning and Individual Differences, 19, 28-33. *

Geary, D. C. (2011). Cognitive predictors of achievement growth in mathematics: A 5-year longitudinal study. Developmental Psychology, 47, 1539-1552.

Gorey, K. M. (2001). Early childhood education: A meta-analytic affirmation of the short- and long-term benefits of educational opportunity. Scho- ol Psychology Quarterly, 16, 9-30. *

Hattie, J. A. C. (2009). Visible learning: A synthesis of over 800 meta-analyses relating to achievement. New York, NY: Routledge.

Hogan, T. P. (2006). Introdução à prática de testes psicológicos. Rio de Janeiro, RJ: LTC.

Horn, J. L. (1994). Theory of fluid and crystallized intelligence. In R. J. Sternberg (Ed.), Encyclopedia of human intelligence (pp. 443-451). New York, NY: Macmillan.

Johnston, A., Gradisar, M., Dohnt, H., Billows, M., \& Mccappin, S. (2010). Adolescent sleep and fluid intelligence performance. Sleep and Biological Rhythms, 8, 180-186. *

Kaufman, A. S., Kaufman, J. C., Liu, X., \& Johnson, C. K. (2009). How do educational attainment and gender relate to fluid intelligence, crystallized intelligence, and academic skills at ages 22-90 years? Archives of Clinical Neuropsychology, 24, 153-163. *

Kuhn, J. T., \& Holling, H. (2009). Gender, reasoning ability, and scholastic achievement: A multilevel mediation analysis. Learning and Individual Differences, 19, 229-233. *

Laros, J. A., Marciano, J. L. P., \& Andrade, J. M. (2010). Fatores que afetam o desempenho na prova de Matemática do SAEB: Um estudo multinível. Avaliação Psicológica, 9, 173-186. *

Laros, J. A., Marciano, J. L. P., \& Andrade, J. M. (2012). Fatores associados ao desempenho escolar em Português: Um estudo multinível por regiões. Ensaio: Avaliação e Políticas Públicas em Educação, 20, 623-646. *

Laros, J. A., Reis, R. F., \& Tellegen, P. J. (2010). Indicações da validade convergente do teste não-verbal de inteligência SON-R 21/2-7[a]. Avaliação Psicológica, 9, 43-52.

Laros, J. A., Tellegen, P. J., Jesus, G. R., \& Karino, C. A. (no prelo). SON-R 21/2-7[a] Teste não-verbal de inteligência: Manual com normatização $e$ validação brasileira. Brasília, DF: Laboratório Meta. *

Leclerc, M., Larivée, S., Archambault, I., \& Janosz, M. (2010). Le sentiment de compétence, modérateur du lien entre le QI et le rendement scolaire en mathématiques. Revue Canadienne de L'Éducation, 33, 31-56. *

Leeson, P., Ciarrochi, J., \& Heaven, P. C. L. (2008). Cognitive ability, personality, and academic per- 
formance in adolescence. Personality and Individual Differences, 45, 630-635. *

McGrew, K. S. (2005). The Cattell-Horn-Carroll theory of cognitive abilities: Past, present, and future. In D. P. Flanagan \& P. L. Harrison (Eds.), Contemporary Intellectual Assessment ( $2^{\text {nd }}$ ed., pp. 136-182). New York, NY: The Guilford Press.

McGrew, K. S., \& Flanagan, D. P. (1998). The intelligence test desk reference (ITDR) - Gc-Gf cross battery assessment. Boston, MA: Allyn and Bacon.

McGrew, K. S., \& Wendling, B. J. (2010). Cattell-Horn-Carroll cognitive-achievement relations: What we have learned from the past 20 years of research. Psychology in the Schools, 47, 651675.

Petrides, K. V., Frederickson, N., \& Furnham, A. (2004). The role of trait emotional intelligence in academic performance and deviant behavior at school. Personality and Individual Differences, 36, 277-293. *

Preckel, F., Lipnevich, A. A., Schneider, S., \& Roberts, R. D. (2011). Chronotype, cognitive abilities, and academic achievement: A meta-analytic investigation. Learning and Individual Differences, 21, 483-492.*

Primi, R. (2003). Inteligência: Avanços nos modelos teóricos e nos instrumentos de medida. Avaliação Psicológica, 1, 67-77.

Primi, R., Ferrão, M. E., \& Almeida, L. S. (2010). Fluid intelligence as a predictor of learning: A longitudinal multilevel approach applied to math. Learning and Individual Differences, 20, 446-451.

Rindermann, H., \& Neubauer, A. C. (2004). Processing speed, intelligence, creativity, and school performance: Testing of causal hypotheses using structural equation models. Intelligence, $32,573-589 . *$

Rohde, T. E., \& Thompson, L. A. (2007). Predicting academic achievement with cognitive ability. Intelligence, 35, 83-92.

Rosander, P., Bäcktröm, M., \& Stenberg, G. (2011).
Personality traits and general intelligence as predictors of academic performance: A structural equation modelling approach. Learning and Individual Differences, 5, 590-596. *

Snijders, J. T., Tellegen, P. J., \& Laros, J. A. (1989). SON-R 51/2-17: Manual and research report. Groningen, Netherlands: Wolters-Noordhoff.

Spearman, C. (1904). "General intelligence” objectively determined and measured. American Journal of Psychology, 15, 201-293.

Steinmayr, R., \& Spinath, B. (2008). Sex differences in school achievement: What are the roles of personality and achievement motivation? European Journal of Personality, 22, 185-209. *

Steinmayr, R., \& Spinath, B. (2009). The importance of motivation as a predictor of school achievement. Learning and Individual Differences, 19, 80-90. *

Steinmayr, R., Ziegler, M., \& Träuble, B. (2010). Do intelligence and sustained attention interact in predicting academic achievement? Learning and Individual Differences, 20, 14-18. *

Swanson, H. L., Jerman, O., \& Zheng, X. (2008). Growth in working memory and mathematical problem solving in children at risk and not at risk for serious math difficulties. Journal of Educational Psychology, 2, 343-379. *

Swartwout, M. D., Garnaat, S. L., Myszka, K. A., Fletcher, J. M., \& Dennis, M. (2010). Associations of ethnicity and SES with IQ and achievement in Spina Bifida Meningomyelocele. Journal of Pediatric Psychology, 35, 927-936. *

Thurstone, L. L. (1938). Primary mental abilities. Chicago, IL: University of Chicago Press. 